\title{
Dolor torácico y shock secundarios a perforación espontánea de esófago: síndrome de Boerhaave
}

\author{
Thoracic pain and shock secondary to spontaneous esophageal perforation: Boherhaave \\ syndrome
}

\author{
Yolanda Núñez-Delgado*, Irene Garrido-Márquez, Laura Díaz-Rubia, Ana Milena-Muñoz, \\ Macarena Eisman-Hidalgo y $M^{a}$ Ángeles Valero-González \\ Servicio de Radiodiagnóstico, Hospital Clínico Universitario San Cecilio, Granada, España
}

\begin{abstract}
Resumen
La perforación esofágica es la más letal de todas las perforaciones del aparato digestivo. Se presenta el caso de un varón de 65 años que acude a urgencias por un cuadro clínico de dolor torácico, vómitos e hipotensión. Se le realizó tomografía computarizada por sospecha de síndrome aórtico agudo, con hallazgos sugerentes de perforación esofágica. El síndrome de Boerhaave consiste en la rotura longitudinal del esófago sobre una pared macroscópicamente sana. Su tratamiento definitivo se realiza con cirugía durante las primeras 24 horas. El síndrome de Boerhaave debe considerarse como complicación posible en los pacientes con dolor epigástrico y vómitos, ya que es una emergencia quirúrgica con alta morbimortalidad.
\end{abstract}

Palabras clave: Dolor torácico. Perforación esofágica espontánea. Síndrome de Boerhaave.

\begin{abstract}
Esophageal perforation is the most lethal of all perforations of the digestive system. 65-year-old male who goes to the emergency department due to clinical symptoms of chest pain, vomiting and hypotension, who underwent CT scan for suspected acute aortic syndrome, with suggestive findings of esophageal perforation. Boerhaave syndrome consists of the longitudinal rupture of the esophagus on a macroscopically healthy wall. Its definitive treatment is performed with surgery during the first 24 hours. Boerhaave syndrome should be considered as a possible complication in patients with epigastric pain and vomiting, as it is a surgical emergency with high morbidity and mortality.
\end{abstract}

Key words: Thoracic pain. Spontaneous esophageal perforation. Boerhaave syndrome.

\section{Introducción}

La perforación espontánea del esófago, también conocida como síndrome de Boerhaave, consiste en la rotura longitudinal del esófago sobre una pared macroscópicamente sana ${ }^{1-3}$. La causa más común de la perforación espontánea es un aumento repentino de la presión dentro de la luz esofágica por una motilidad descoordinada durante un vómito intenso $0^{1,2}$, aunque también puede ocurrir durante el parto, por convulsiones, tos o risa prolongada. Se suele romper la pared esofágica posterolateral izquierda a unos 2-3 
cm de la unión gastroesofágica en el 90\% de los casos. Es la causa más grave de perforación esofágica y presenta una mortalidad muy elevada ${ }^{2}$.

\section{Caso clínico}

Varón de 65 años, con antecedentes de asma y cáncer de próstata, que acude a urgencias por cuadro de vómitos, dolor centro-torácico e hipotensión de 4 horas de evolución. Se le realizó tomografía computarizada (TC) con contraste iodado intravenoso, por sospecha clínica de síndrome aórtico agudo, y se observó una gran colección líquida con gas en la región del hiato esofágico, que sugería perforación a ese nivel (Fig. 1). El paciente fue intervenido mediante laparotomía media supraumbilical, encontrándose una perforación de unos $3 \mathrm{~cm}$ en la cara posterolateral izquierda del esófago distal, a nivel del hiato esofágico, con mediastinitis y peritonitis secundarias, así como rotura de la curvatura menor gástrica. Se realizó cierre primario de la perforación con exclusión esofágica cervical y drenaje mediastínico de la colección y sonda de yeyunostomía. Una TC realizada a las 24 horas de la cirugía, por mala evolución clínica, puso de manifiesto una perforación yatrogénica de 6 $\mathrm{cm}$ en pared traqueal posterior, probablemente durante la intubación, que fue tratada mediante toracotomía posterolateral, reparación primaria y mioplastia de serrato mayor, dejando tres sondas de pleurostomía.

La evolución fue favorable, siendo dado de alta hospitalaria a las 4 semanas de su ingreso. En una revisión a los 3 meses, el paciente se encontraba asintomático desde el punto de vista digestivo, tolerando sólidos y sin disfagia.

\section{Discusión}

La perforación esofágica es la más seria y letal de todas las perforaciones del aparato digestivo ${ }^{1-3}$. Es poco frecuente. Se define como una dehiscencia de la pared esofágica en una zona macroscópicamente sana, en general a consecuencia de un aumento brusco de la presión esofágica, en combinación con una presión intratorácica negativa causada por los vómitos o esfuerzos ${ }^{3}$. La mayoría de los casos ocurren en pacientes con un esófago subyacente normal, aunque la presencia de esofagitis o de úlceras también se ha encontrado en algunos individuos.

La anamnesis y la exploración física suelen ser de poca ayuda en el diagnóstico. El retraso en el diagnóstico ocurre hasta en un $50 \%$ de los pacientes,

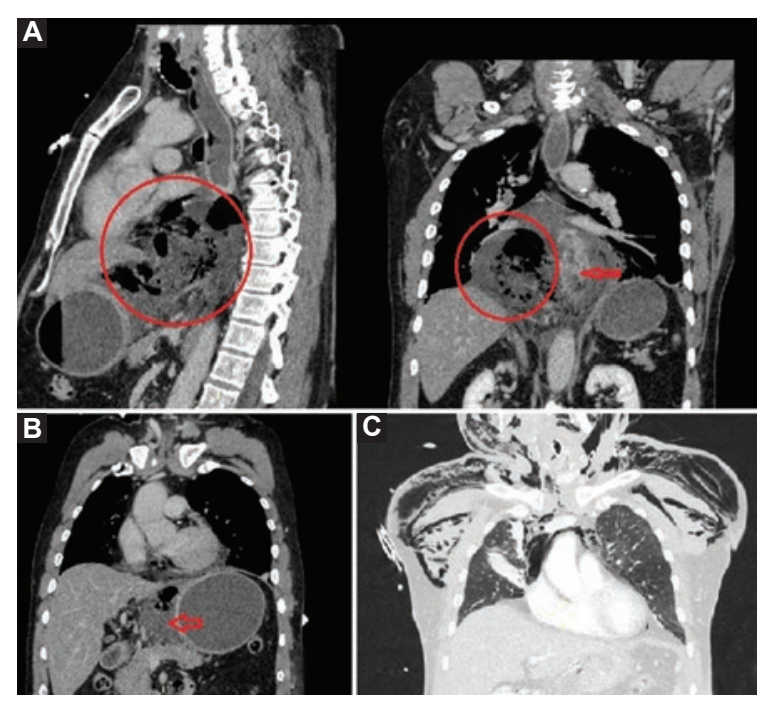

Figura 1. A: tomografía computarizada torácica con contraste intravenoso yodado en ventana de mediastino, plano sagital, derecha, $y$ plano coronal, izquierda. Gran colección líquida con abundante contenido aéreo intratorácica (círculos). Hipercaptación de contraste de la mucosa esofágica (flecha). B: colección líquida con gas en espacio gastrohepático, en relación con peritonitis. C: tomografía computarizada de tórax en ventana de pulmón, plano coronal. Enfisema subcutáneo y neumomediastino grave secundario a rotura yatrogénica de tráquea.

debido a la inespecificidad de los síntomas. Los síntomas más habituales son náuseas y vómitos graves, seguidos de dolor agudo retroesternal, en ocasiones irradiado al hombro izquierdo, y abdominal alto, que se siguen rápidamente de disnea y shock. Por tanto, debe sospecharse cuando se asocian síntomas digestivos y respiratorios. Es muy característica la tríada de Mackler: vómitos, dolor torácico y enfisema subcutáneo; no obstante, esta aparece en muy pocas ocasiones ${ }^{3}$.

La primera prueba de imagen que debe realizarse es la radiografía simple, que es normal en aproximadamente el $15 \%$ de los casos ${ }^{4}$. Si existen alteraciones radiológicas iniciales secundarias a la perforación, las más frecuentes son el neumomediastino y el neumoperitoneo, pudiendo aparecer horas o días después derrame pleural con o sin neumotórax, ensanchamiento mediastínico y enfisema subcutáneo. En los pacientes con clínica y radiografía de tórax sospechosas se solicitará una TC o un esofagograma, o ambos. En la TC pueden visualizarse colecciones líquidas o niveles hidroaéreos periesofágicos sugestivos de perforación, como en nuestro caso, pero habitualmente no determina la localización ni el tamaño de la perforación, lo que se hace con el esofagograma con contraste hidrosoluble, que tiene una 
especificidad y una sensibilidad cercanas al $100 \%$. La realización de la esofagoscopia sigue siendo polémica, ya que la insuflación de aire puede aumentar el tamaño de la lesión y pasar aire al mediastino ${ }^{3}$.

En nuestro caso, los hallazgos en la TC sugerían el diagnóstico y fue suficiente para intervenir quirúrgicamente de forma urgente al paciente.

El diagnóstico diferencial hay que hacerlo con múltiples patologías, entre ellas la pancreatitis, el infarto agudo de miocardio, la disección de aorta, la úlcera perforada, la embolia pulmonar y la neumonía por aspiración.

Una revisión reciente propone el diagnóstico de los «big five», que amenazan la vida del paciente y que habría que investigar en la sala de emergencias: síndrome coronario agudo, embolia pulmonar aguda, síndrome aórtico agudo, neumotórax a tensión y síndrome de Boerhaave ${ }^{6}$.

El síndrome de Boerhaave se asocia con una alta mortalidad $^{1-3}$. Los principales factores pronósticos son el tamaño y la localización de la perforación, y la demora en el diagnóstico, con la consecuente contaminación de la pleura y el mediastino ${ }^{3}$.

El tratamiento conservador se realizará en las siguientes situaciones, según los criterios de Cameron de 1979 reformados por Altorjay en 1997: perforaciones intramurales, perforaciones detectadas de forma precoz o circunscritas si se han detectado tardíamente, perforaciones transmurales que no estén ubicadas en el abdomen y que estén contenidas en el mediastino y drenando al esófago, aquellas que no estén asociadas a obstrucción esofágica o cáncer, y en pacientes con síntomas mínimos y sin evidencia clínica de sepsis?.

En cambio, la reparación quirúrgica durante las primeras 24 horas es la mejor opción, aunque se asocia con una alta tasa de morbimortalidad. En los últimos años han aparecido técnicas endoscópicas para resolver la perforación gastrointestinal, como la EVAC (Endoscopic vacuum-assisted closure), con una tasa de éxito del $95-100 \%{ }^{8,9}$. Con respecto al tratamiento endoscópico, se pueden proponer diferentes mecanismos terapéuticos: derivación con stentsautoexpandibles; cierre con OTSC (Over-The-Scope Clip), que podría usarse para perforación gastrointestinal $\leq 10 \mathrm{~mm}^{10}$; y terapias locales que consisten en lavado, antibióticos locales y drenaje interno de la colección. Los stents metálicos autoexpandibles han sido ampliamente evaluados para su uso en casos de fístulas y fugas esofágicas, ayudando a evitar la cirugía en el $60 \%$ de los pacientes en la primera serie publicada y mostrando un $100 \%$ de éxito más recientemente ${ }^{9}$.

\section{Conflicto de intereses}

Los autores declaran no tener ningún conflicto de intereses.

\section{Financiamiento}

Los autores declaran que para esta investigación no se ha recibido ningún financiamiento.

\section{Responsabilidades éticas}

Protección de personas y animales. Los autores declaran que para esta investigación no se han realizado experimentos en seres humanos ni en animales.

Confidencialidad de los datos. Los autores declaran que han seguido los protocolos de su centro de trabajo sobre la publicación de datos de pacientes.

Derecho a la privacidad y consentimiento informado. Los autores han obtenido el consentimiento informado de los pacientes y/o sujetos referidos en el artículo. Este documento obra en poder del autor de correspondencia.

\section{Bibliografía}

1. Rokicki M, Rokicki W, Moj M, Bsoul T, Rydel M. Boerhaave syndrome - over 290 years of surgical experiences. Pol Przegl Chir. 2018;91:27-9.

2. García Calleja JL, Ferrer Sotelo R, Lagunas Lostao E, Borlán-Ansón S, Valero-Sabater M, Fernando-Trebolle J, et al. Perforación esofágica espontánea o síndrome de Boerhaave. Cir Esp. 2015;93 (Espec Congr):422.

3. Pérez Villaverde N, Soto García M, Alemán Villanuevac S, Barrios Barbero A, Rodríguez Jiménez S. Síndrome de Boerhaave. Rev Clin Med Fam. 2015;8:158-61.

4. Turner AR, Turner SD. Boerhaave syndrome. Treasure Island (FL): StatPearls Publishing; 2020.

5. Granados Mora E. Síndrome de Boerhaave: generalidades y manejo basado en la evidencia. Revista Médica de Costa Rica y Centroamérica 2015;72:361-6.

6. Fitzpatrick D, Longondjo M. Gastric perforation through a hiatus hernia into the left lung in an 84-year-old woman. BMJ Case Rep. 2019; 12:e227956.

7. Braghetto I, Rodríguez NA, Csendes A, Korn O. Perforación esofágica: experiencia clínica y actualización del tema. Revista Médica de Chile. 2005;133: 1233-41.

8. Min YW, Kim T, Lee H, Min BH, Kim HK, Choi YS, et al. Endoscopic vacuum therapy for postoperative esophageal leak. BMC Surg. 2019; 19:37.

9. Tellechea JI, González J-M, Miranda-García P, Culetto A, Benoit D, Journo X, et al. Role of endoscopy in the management of Boerhaave syndrome. Clin Endosc. 2018;51:186-91.

10. Núñez Rodríguez MH, Sánchez Martín F, Nájera R, Díez Redondo P. Over-the-scope-clip: endoscopic treatment of Boerhaave syndrome. Gastroenterol Hepatol. 2019;42:564-5. 